\title{
Effects of Agricultural Practice on Bird Species Composition in Ilejemeje Local Government Area of Ekiti State, Nigeria
}

\section{Ogunyemi 00*}

Department of Forest Resources and Wildlife Management, Nigeria

*Corresponding author: Ogunyemi 00, Department of Forest Resources and Wildlife Management, Ekiti State University, Ado-Ekiti, Nigeria, Email: olumideogunyemi80@yahoo. com

\section{Review Article}

Volume 4 Issue 4

Received Date: May 25, 2020

Published Date: June 23, 2020

DOI: $10.23880 /$ jenr-16000203

\section{Abstract}

The study was carried out in Ilejemeje local government area of Ekiti State, from July - November 2018, for the comparative evaluation of avifauna abundance and diversity of the three (3) prominent land use types of the area (Maize, Cocoa and Natural forest). A total of thirty -eight (38) bird species belonging to thirteen (13) order and twenty-one (21) families inhabits the land use types of Ilejemeje local government area of Ekiti State . Maize plantation land use type contained a greater number of bird species, than the other two land use types. On the overall a total of one thousand, five hundred and thirty-one $(1,531)$ individual bird species were distributed within the land use types. The abundance of bird species vary with the three land use types, the maize plantation recorded the highest percentage value of $41.61 \%$ of individual bird species followed by the natural forest $38.83 \%$ while Cocoa plantation recorded the least percentage value of $27.56 \%$. The community structure of bird trophic guilds in the study area revealed that granivore-insectivore, frugivore-insectivore and insectivore were the prominent trophic guilds group in the three land use types. Maize plantation had the highest species diversity and richness while the Cocoa plantation had the highest species evenness. The relative abundance score of species during the study showed that most of the birds observed were in frequent abundant categories.

Keywords: Granivore-insectivore; Frugivore-insectivore; Insectivore

\section{Introduction}

Habitat loss and numerous undesirable environmental disturbances associated with agricultural practices has spur up conservation scientists to conclude that agriculture is one of the major contributors towards biodiversity loss Sala [1]. Musters [2] asserted that roughly one-third of the world land area has been taken over by agricultural system, while Tilman [3] reported that the world's demand for food is expected to be twice the present trend in demand, in view of which more land area is expected to be cleared for agricultural practices.

Conservation in the tropics has it focus and attention on natural, undisturbed habitats like rainforest [4], while lesser attention is given to agricultural landscape in ecological and conservation research [5]. With the advancement of civilization the natural habitats has been decreasing in area and reasonable number of species of wild creatures have no alternative than to depend in the usage of land heavily used by man and the types, scale of agriculture system being practiced have been discovered to have a significant role to play on wild creatures populations whose ecosystem fall within the area [6,7]. As the rate of clearing of pristine habitat for agricultural practices increases, survival of diversity of species that invariably depend on them either decreases or increases and that the patterns of use, the types of crops planted in agricultural landscapes determine the extent of species diversity, this necessitated that the effect of various patterns of agricultural land use by wild species population 


\section{Journal of Ecology and Natural Resources}

must be given adequate study in order to incorporate them into conservation management plans. The future increase in agricultural areas has been predicted to be mostly concentrated in the lowland rainforest, where the majority of planet's most important conservation areas are located $[8,9]$. One can therefore infer that the pertinent challenges with which nature conservationist will be confronted within the nearest future will be the discovering the correlation existing between the changing environments and how agriculture as a major driving force in the alteration of pristine ecosystems can be managed effectively to accommodate both human needs and biodiversity conservation. Hence there is a great need to establish the contribution of various forms of agricultural system on the sustainability of biodiversity.

\section{Study Areas}

The study was carried out in Ilejemeje local government area of Ekiti state. Ekiti state lies within the tropic and located between $4^{0} 5^{5^{\prime}}$ to $5^{0} 4^{5^{\prime}}$ East of Greenwich meridian and latitude $7^{0} 15^{\prime}$ to $8^{0} 15^{\prime}$ North of the equator (Figure 1). The climate of the state is tropical, with average temperature of $25^{\circ} \mathrm{C}$ all year round and high relative humidity. The rainy season has an average of 270 days. The annual average rainfall varies from about $2500 \mathrm{~mm}$ to $4000 \mathrm{~mm}$ across the state. The vegetation of the state is diverse and has been described by several authors to fall into forest eco-climate vegetation. The study area (Ilejemeje local government area) is characterized by secondary forests and agricultural land use systems with mainly cocoa, kolanut, cassava, yam and maize as major crops. Within this landscape, study sites were selected in three different land-use-types: maize plantation (MP), cocoa plantation (CP) and natural secondary forest (NF) representing the full range of land-use types in the area.

The study sites were located in four (4) towns: Iye, Ewu, Iludun and Ijeshamodu which were randomly selected out of seven (7) towns that constitutes the local government area. Two replicates points were selected from each of the three studied land-use types, Maize plantation (MP. $\left.\mathrm{P}_{1}, \mathrm{P}_{2}\right)$, Cocoa plantation $\left(C P . \mathrm{P}_{1}, \mathrm{P}_{2}\right)$ and Natural forest $\left(N F . \mathrm{P}_{1}, \mathrm{P}_{2}\right)$.

\section{Method of Data Collection}

A survey of abundance and diversity of Avifauna species associated with the selected three land-use types of Ilejemeje local government area of Ekiti State was conducted between the months of July to November, 2018. Line transects count method as described by Billy [10] was employed for the collection of data on bird species diversity and abundance in the sample sites. The method involved the location of two lines transect of $1 / 2 \mathrm{~km}$ lengths in each of the land use types.

Birds were then observed by walking along the established transects in each of the land use types for three consecutive days. Following Jones [11] data collection commenced about 30 minutes after dawn and was carried out for five hours daily from $6.30-10.00 \mathrm{am}$ in the morning and $4.30-6.00 \mathrm{pm}$ in the evening when the activities of birds were prominent. To avoid repeated counting of birds, transects established were reasonably spaced out by $500 \mathrm{~m}$ distance apart.

In all transects, the relative value of each the three defined land use types for attracting different bird species was recorded through direct observation. The birds were visually observed with the aid of binocular and their taxonomic groups were properly categorized based on field guides to birds of western Africa [12].

\section{Data Analysis}

The data collected from varying land use types of Ilejemeje local government area of Ekiti State were put together to determine the species composition, and abundance of bird species, the percentage of each birds were determined. The data were further subjected to descriptive statistics (frequency distribution and histogram) species richness index, Shannon wiener's index and species evenness index using the following formulas.

Shannon Wiener diversity index is given by

$$
\mathrm{H} 1=-\sum \text { pi } \ln (\mathrm{p})
$$

Where: $\mathrm{H}=$ Shannon Wiener diversity index

$\mathrm{S}=$ total number of bird species in the community

$\mathrm{P}_{\mathrm{i}}=$ Proportion of birds in the species

In = natural logarithms

$\Sigma=$ summation

Evenness index (E)

$$
\mathrm{E}=\frac{\mathrm{H}^{1}}{\log \mathrm{S}}
$$

Where

$\mathrm{H}^{1}$ shannon - Wiener's diversity index

$\mathrm{S}$ - Species richness

Species richness (D)

$$
\mathrm{D}=\frac{\mathrm{S}-1}{\log \mathrm{N}}
$$

where $\mathrm{S}=$ Number of Species

$\mathrm{N}=$ Number of individual,

\section{Results}

The result of this study has shown that a total of thirty eight (38) bird species belonging to thirteen (13) orders 
and twenty- one (21) families inhabits the land use types of Ilejemeje local government area of Ekiti State. Table 1, maize plantation (MP) land use type contained significantly a greater number of bird species than the other two land use types. Indeed, of the thirty eight (38) species recorded, the highest number of bird species across the land use types were recorded in maize plantation (34) compare to (22) in cocoa plantation (CP) and (25) in natural forest (NF) (Table 2). The order Passeriformes constituted the predominant group representing $42.86 \%$ of families $(n=09)$ and $42.11 \%$ of species $(n=16)$. The family with the largest number of species were Ploceidae $(n=6)$ and Accipitridae $(n=5)$. Six bird species, such as Black Kite (Milvus migrans), Double spurred francolin (Francolinus bicalcaratus), Slender-billed weaver (Ploceus pelzeli), Hunter Sunbird (Nectarinia hunteri), Africa Trush (Turchus pelios), Little swift (Apus affinis) White wing starling (Neocichia gutturdis) ranked highest in frequency of occurrence in the three land use types (Table 6).

A total of 1,531 individual bird species were counted during the study period. The individual bird species were distributed within the land use types namely maize plantation (MP), cocoa plantation (CP) and natural forest (NF) of Ilejemeje Local Government Area of Ekiti State. The maize plantation recorded the highest percentage value of $41.61 \%$ of individual bird species followed by the natural forest which recorded $30.83 \%$ while cocoa plantation recorded the least percentage value of $27.56 \%$ of individual bird species (Table 3 ). Table 2 vividly shows the variation that exists between orders, families and species in the three land use types of the study area. Exclusive bird species were more prevalent in the maize plantation with $42.86 \%(\mathrm{n}=12)$ (Table 2$)$. The analysis of the relative abundance of the birds observed in the land use types of the local government area are shown in Figure 3 indicated that of the bird species inhabiting the area five (5) bird species were uncommon, nineteen (19) bird species were frequent, fourteen (14) species were common, while none of the bird species observed were in rare and abundant categories.

The diversity and evenness analysis was carried out based on the number of individuals of bird species in the three land use types and results is presented in Table 3 . When the Shannon Weiner diversity index $\left(\mathrm{H}^{1}\right)$ and species richness across the three land use types is considered, it showed that the maize plantation with index values that ranges from 0.83- 0.89 and 2.78- 4.02 respectively had the highest species diversity and richness, followed by natural forest, with values range of 0.68- 0.88 and 2.01- 3.52 respectively, while cocoa plantation had the least species diversity and richness with the index values $0.59-0.86$ and $1.62-3.64$ respectively. With the regard to species evenness across the land use types, cocoa plantation registered more even distribution of bird species during the study, followed by natural plantation.

\begin{tabular}{|c|c|c|c|c|}
\hline S/NO & Species & Scientific Names & FAMILY & ORDER \\
\hline $\mathbf{1}$ & Black kite & Milvus migrans & Acciptridae & Falconiformes \\
\hline $\mathbf{2}$ & Hooded vulture & Necrosyrtes monachus & Acciptridae & Falconiformes \\
\hline $\mathbf{3}$ & Harrier hawk & Polyboroides radiates & Acciptridae & Falconiformes \\
\hline $\mathbf{4}$ & Tawny eagle & Aquil rapax & Acciptridae & Falconiformes \\
\hline $\mathbf{5}$ & Shikra & Accipiter badius & Acciptridae & Falconiformes \\
\hline $\mathbf{6}$ & Kestrel & Falco tinnunculus & Falconidae & Falconiformes \\
\hline $\mathbf{7}$ & Double spurred francolin & Fancolinus bicalcaratus & Phasianidae & Galliformes \\
\hline $\mathbf{8}$ & Red-billed dward hornbill & Tockus camurus & Bucerotidae & Coraciformes \\
\hline $\mathbf{9}$ & Black-casqued hornbill & Ceratogymna atrata & Bucerotidae & Coraciformes \\
\hline $\mathbf{1 0}$ & Black and white-casqued hornbill & Bycanistes subcylindricus & Bucerotidae & Coraciformes \\
\hline $\mathbf{1 1}$ & Green wood hoopoe & Phoeniculus purpureus & Upupidae & Coraciformes \\
\hline $\mathbf{1 2}$ & Red-billed hornbill & Tockus erythrorhynchus & Bucerotidae & Coraciformes \\
\hline $\mathbf{1 3}$ & White throated bee-eater & Merops albicollis & Meropidae & Coraciformes \\
\hline $\mathbf{1 4}$ & Dwarf kingfisher & Ispidina lecontei & Alcedinidae & Coraciformes \\
\hline $\mathbf{1 5}$ & Black heron & Egretta ardesiaca & Ardeidae & Ciconiiformes \\
\hline $\mathbf{1 6}$ & Woolly-necked stork & Ciconia episcopus & Ciconiidae & Ciconiiformes \\
\hline $\mathbf{1 7}$ & Narinas trogon & Apaloderma narina & Trogonidae & Ciconiiformes \\
\hline $\mathbf{1 8}$ & African thrush & Turdus pelios & Turdidae & Passeriformes \\
\hline $\mathbf{1 9}$ & Laughing dove & Streptopelia senegalensis & Columbidae & Columbiformes \\
\hline $\mathbf{2 0}$ & Red-neck dove & Streptopelia capicola & Columbidae & Columbiformes \\
\hline $\mathbf{2 1}$ & Lemon dove & Aplopelia larvata & Columbidae & Columbiformes \\
\hline
\end{tabular}




\begin{tabular}{|c|c|c|c|c|}
\hline $\mathbf{2 2}$ & Little swift & Apus afinis & Apodidae & Apodiformes \\
\hline $\mathbf{2 3}$ & Mottled swift & Tachymarptis aequatorialis & Apodidae & Apodiformes \\
\hline $\mathbf{2 4}$ & Hunter sunbird & Nectarinia hunter & Nectoriniidae & Passeriformes \\
\hline $\mathbf{2 5}$ & White crowned starling & Spreo albicapillus & Sturnidae & Passeriformes \\
\hline $\mathbf{2 6}$ & Square-taled drongo & Dicrurus ludwigii & Dicruridae & Passeriformes \\
\hline $\mathbf{2 7}$ & Slender- billed weaver & Ploceus pelzeini & Ploceidae & Passeriformes \\
\hline $\mathbf{2 8}$ & Black-billed water & Ploceus cucullatus & Ploceidae & Passeriformes \\
\hline $\mathbf{2 9}$ & Chestnut weaver & Ploceus ribiginosus & Ploceidae & Passeriformes \\
\hline S/NO & Species & Scientific Names & FAMILY & ORDER \\
\hline $\mathbf{3 0}$ & Red handed weaver & Anaplectes rubriceps & Ploceidae & Passeriformes \\
\hline $\mathbf{3 1}$ & Compact weaver & Ploceus pachyrhnchus & Ploceidae & Passeriformes \\
\hline $\mathbf{3 2}$ & Red-chested swallow & Hirundo lucida & Hirundinidae & Passeriformes \\
\hline $\mathbf{3 3}$ & Red-rumped swallow & Cecropis daurica & Hirundinidae & Passeriformes \\
\hline $\mathbf{3 4}$ & Cape rook & Corvus capensis & Corvidae & Passeriformes \\
\hline $\mathbf{3 5}$ & White wing starling & Neocichla gutturalis & Sturnidae & Passeriformes \\
\hline $\mathbf{3 6}$ & Fisher's starling & Spreo fischeri & Sturnidae & Passeriformes \\
\hline $\mathbf{3 7}$ & Senegal coucal & Serinus atrogularis & Cuculidae & Cuculiformes \\
\hline $\mathbf{3 8}$ & Yellow-rumped seed-eater & Cuculiformes \\
\hline
\end{tabular}

Table 1: Avian distribution based on family, order and species composition in the three land use types of Ilejemeje local government area Ekiti State.

Table 4 shows the trophic guilds of bird species in the three land use types of Ilejemeje local government area of Ekiti State. The different feeding guilds existing in the study area has been shown to vary among the different land use types. In terms of community structure of bird trophic guilds, granivore-Insectivore (GI), frugivore-insectivore (FI) and insectivore (I) were the predominant trophic guilds group in the three land use types

\begin{tabular}{|c|c|c|c|c|c|c|c|c|c|c|c|c|}
\hline & \multicolumn{3}{|c|}{ Orders } & \multicolumn{3}{|c|}{ Families } & \multicolumn{3}{|c|}{ No. Of Species } & \multicolumn{3}{|c|}{ No Of Exclusive Species } \\
\hline 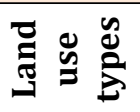 & MP & $\mathrm{CP}$ & NF & MP & $\mathbf{C P}$ & NF & MP & $\mathbf{C P}$ & NF & MP & $\mathbf{C P}$ & NF \\
\hline I & 06 & 04 & 02 & 07 & 08 & 04 & 10 & 09 & 07 & 02 & 02 & 03 \\
\hline $\mathbf{E}$ & 06 & 03 & 07 & 09 & 04 & 07 & 09 & 04 & 08 & 03 & 01 & 02 \\
\hline IL & 04 & 02 & 04 & 04 & 04 & 03 & 07 & 04 & 05 & 03 & 02 & 02 \\
\hline IJ & 07 & 03 & 03 & 07 & 04 & 05 & 08 & 05 & 05 & 04 & 02 & 02 \\
\hline
\end{tabular}

Table 2: Orders, families and no of bird species observed in the three land-use types of Ilejemeje local government area.

\begin{tabular}{|c|c|c|c|c|c|c|c|c|c|c|c|c|}
\hline \multirow[t]{2}{*}{ Location } & \multicolumn{3}{|c|}{ I } & \multicolumn{3}{|c|}{$\mathbf{E}$} & \multicolumn{3}{|c|}{ IL } & \multicolumn{3}{|c|}{ IJ } \\
\hline & MP & $\mathbf{C P}$ & NF & MP & $\mathbf{C P}$ & NF & MP & $\mathbf{C P}$ & NF & MP & $\mathbf{C P}$ & NF \\
\hline No of Bird species & 10 & 09 & 07 & 09 & 04 & 08 & 07 & 04 & 05 & 08 & 05 & 05 \\
\hline $\begin{array}{c}\text { Total No of individual Bird } \\
\text { species }\end{array}$ & 172 & 159 & 143 & 186 & 64 & 149 & 145 & 73 & 98 & 134 & 126 & 82 \\
\hline Species richness & 4.02 & 3.64 & 2.78 & 3.52 & 1.66 & 3.23 & 2.78 & 1.62 & 2.01 & 3.29 & 1.90 & 2.09 \\
\hline Shannon diversity $\left(\mathrm{H}^{1}\right)$ & 0.89 & 0.86 & 0.82 & 0.89 & 0.60 & 0.88 & 0.82 & 0.59 & 0.69 & 0.83 & 0.70 & 0.68 \\
\hline Evenness & 0.87 & 1.39 & 0.92 & 0.89 & 1.46 & 0.98 & 0.89 & 0.99 & 0.97 & 0.94 & 0.99 & 0.95 \\
\hline
\end{tabular}

Table 3: Species Richness, Diversity Index and Evenness of Bird Species in Three Land Use Types of Ilejemeje Local Government Area. 


\begin{tabular}{|c|c|c|}
\hline Common Names & Scientific Names & Trophic guilds \\
\hline Black kite & Milvus migrans & 0 \\
\hline Hooded vulture & Necrosyrtes monachus & 0 \\
\hline Harrier hawk & Polyboroides radiates & $\mathrm{C}$ \\
\hline Tawny eagle & Aquil rapax & $\mathrm{C}$ \\
\hline Shikra & Accipiter badius & $\mathrm{C}$ \\
\hline Kestrel & Falco tinnunculus & $\mathrm{Cl}$ \\
\hline Double spurred francolin & Fancolinus bicalcaratus & $\mathrm{Cl}$ \\
\hline Red-billed dward hornbill & Tockus camurus & $\mathrm{Fl}$ \\
\hline Black-casqued hornbill & Ceratogymna atrata & $\mathrm{Fl}$ \\
\hline Black and white-casqued hornbill & Bycanistes subcylindricus & $\mathrm{Fl}$ \\
\hline \begin{tabular}{|l} 
Green wood hoopoe \\
\end{tabular} & Phoeniculus purpureus & $\mathrm{I}$ \\
\hline Red-billed hornbill & Tockus erythrorhynchus & $\mathrm{Fl}$ \\
\hline White throated bee-eater & Merops albicollis & $\mathrm{I}$ \\
\hline Dwarf kingfisher & Ispidina lecontei & $\mathrm{Pl}$ \\
\hline Black heron & Egretta ardesiaca & $\mathrm{CP}$ \\
\hline Woolly-necked stork & Ciconia episcopus & $\mathrm{Cl}$ \\
\hline Narinas trogon & Apaloderma narina & $\mathrm{I}$ \\
\hline African thrush & Turdus pelios & $\mathrm{Fl}$ \\
\hline Laughing dove & Streptopelia senegalensis & GI \\
\hline Red-neck dove & Streptopelia capicola & GI \\
\hline Lemon dove & Aplopelia larvata & GI \\
\hline Little swift & Apus afinis & $\mathrm{I}$ \\
\hline Mottled swift & Tachymarptis aequatorialis & $\mathrm{I}$ \\
\hline Hunter sunbird & Nectarinia hunter & IN \\
\hline White crowned starling & Spreo albicapillus & $\mathrm{Fl}$ \\
\hline Square-taled drongo & Dicrurus ludwigii & $\mathrm{I}$ \\
\hline Slender- billed weaver & Ploceus pelzeini & $\mathrm{Gl}$ \\
\hline Black-billed water & Ploceus cucullatus & $\mathrm{Gl}$ \\
\hline Chestnut weaver & Ploceus ribiginosus & Gl \\
\hline Red handed weaver & Anaplectes rubriceps & $\mathrm{Gl}$ \\
\hline Compact weaver & Ploceus pachyrhnchus & Gl \\
\hline Red-chested swallow & Hirundo lucida & $\mathrm{I}$ \\
\hline Red-rumped swallow & Cecropis daurica & $\mathrm{I}$ \\
\hline Cape rook & Corvus capensis & 0 \\
\hline White wing starling & Neocichla gutturalis & $\mathrm{Fl}$ \\
\hline Fisher's starling & Spreo fischeri & FI \\
\hline Senegal coucal & Centropus senegalensis & $\mathrm{Cl}$ \\
\hline Yellow-rumped seed-eater & Serinus atrogularis & $\mathrm{Gl}$ \\
\hline
\end{tabular}

Table 4: Feeding guilds of bird species observed in the three land use types of Ilejemeje local government area.

$\mathrm{O}=$ Omnivores, $\mathrm{C}=$ Carnivores, $\mathrm{Cl}=$ Carnivores/Insectivores, $\mathrm{I}=$ Insectivores, $\mathrm{PI}=$ Piscivores/Insectivores, $\mathrm{CP}=\mathrm{Carnivores} /$ Piscivores, FI= Frugivores/Insectivores, GI= Granivores/Insectivores, IN= Insectivores/Nectivores 


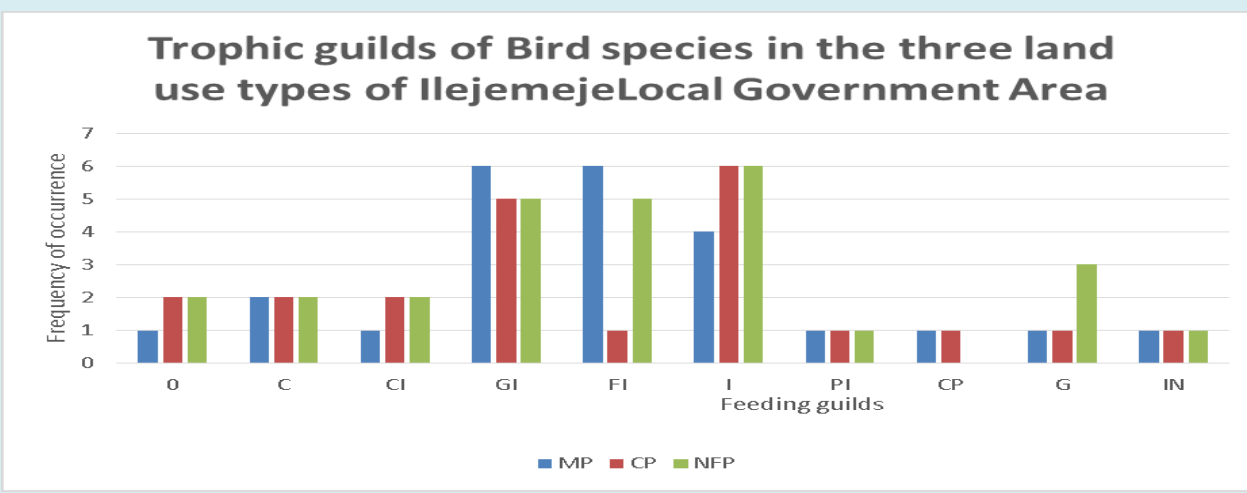

Figure 2: Trophic guilds of Bird species in the three land use types of Ilejemeje L G A.

\begin{tabular}{|c|c|c|c|c|}
\hline & Scientific Names & MP & $\mathbf{C P}$ & NF \\
\hline Black Kite & Milvus migrans & + & + & + \\
\hline Hooded Vulture & Necrosyrtes monachus & - & + & + \\
\hline Harrier Hawk & Polyboroides radiates & + & + & + \\
\hline Tawny Eagle & Aquil rapax & + & + & + \\
\hline Shikra & Accipiter badius & + & - & - \\
\hline Kestrel & Falco tinnunculus & - & - & + \\
\hline Double Spurred Francolin & Fancolinus bicalcaratus & - & - & + \\
\hline Red-Billed Dward Hornbill & Tockus camurus & + & - & + \\
\hline Black-Casqued Hornbill & Ceratogymna atrata & + & - & + \\
\hline Black And White-Casqued Hornbill & Bycanistes subcylindricus & + & - & + \\
\hline Green Wood Hoopoe & Phoeniculus purpureus & - & + & + \\
\hline Red-Billed Hornbill & Tockus erythrorhynchus & + & - & + \\
\hline White Throated Bee-Eater & Merops albicollis & - & + & - \\
\hline Dwarf Kingfisher & Ispidina lecontei & + & + & + \\
\hline Black Heron & Egretta ardesiaca & + & + & - \\
\hline Woolly-Necked Stork & Ciconia episcopus & - & + & - \\
\hline Narinas Trogon & Apaloderma narina & - & + & - \\
\hline African Thrush & Turdus pelios & - & + & - \\
\hline Laughing Dove & Streptopelia senegalensis & + & + & + \\
\hline Red-Neck Dove & Streptopelia capicola & - & - & + \\
\hline Lemon Dove & Aplopelia larvata & - & - & + \\
\hline Little Swift & Apus afinis & + & + & + \\
\hline Mottled Swift & Tachymarptis aequatorialis & + & + & + \\
\hline Hunter Sunbird & Nectarinia hunter & + & + & + \\
\hline White Crowned Starling & Spreo albicapillus & + & - & + \\
\hline Square-Taled Drongo & Dicrurus ludwigii & + & + & + \\
\hline
\end{tabular}




\begin{tabular}{|c|c|c|c|c|}
\hline Slender- Billed Weaver & Ploceus pelzeini & + & + & + \\
\hline Black-Billed Water & Ploceus cucullatus & + & + & + \\
\hline Chestnut Weaver & Ploceus ribiginosus & + & + & + \\
\hline Red Handed Weaver & Anaplectes rubriceps & + & + & + \\
\hline Compact Weaver & Ploceus pachyrhnchus & + & + & + \\
\hline Red-Chested Swallow & Hirundo lucida & + & - & + \\
\hline Red-Rumped Swallow & Cecropis daurica & + & + & + \\
\hline Cape Rook & Corvus capensis & - & - & + \\
\hline White Wing Starling & Neocichla gutturalis & + & - & + \\
\hline Fisher's Starling & Spreo fischeri & - & - & + \\
\hline Senegal Coucal & Centropus senegalensis & + & + & + \\
\hline Yellow-Rumped Seed-Eater & Serinus atrogularis & + & - & + \\
\hline
\end{tabular}

Table 5: Bird Species Distribution of the Three Land Use Types of Ilejemeje Local Government Area.

\begin{tabular}{|c|c|c|c|c|c|}
\hline$S / N$ & Species & Scientific Names & No Observed & Percent & Rel. Abundance \\
\hline 1 & Black kite & Milvus migrans & 85 & $5.55 \%$ & 0.06 \\
\hline 2 & Hooded vulture & Necrosyrtes monachus & 10 & $0.65 \%$ & 0.01 \\
\hline 3 & Harrier hawk & Polyboroides radiates & 26 & $1.70 \%$ & 0.02 \\
\hline 4 & Tawny eagle & Aquila rapax & 17 & $1.11 \%$ & 0.01 \\
\hline 5 & Shikra & Accipiter badius & 52 & $3.40 \%$ & 0.03 \\
\hline 6 & Kestrel & Falco tinnunculus & 21 & $1.37 \%$ & 0.01 \\
\hline 7 & Double spurred francolin & Fancolinus bicalcaratus & 96 & $6.27 \%$ & 0.06 \\
\hline 8 & Red-billed dward hornbill & Tockus camurus & 18 & $1.18 \%$ & 0.01 \\
\hline 9 & Black-casqued hornbill & Ceratogymna atrata & 93 & $6.07 \%$ & 0.06 \\
\hline 10 & $\begin{array}{l}\text { Black and white-casqued } \\
\text { hornbill }\end{array}$ & Bycanistes subcylindricus & 19 & $1.24 \%$ & 0.01 \\
\hline 11 & Green wood hoopoe & Phoeniculus purpureus & 16 & $1.05 \%$ & 0.01 \\
\hline 12 & Red-billed hornbill & Tockus erythrorhynchus & 53 & $3.46 \%$ & 0.03 \\
\hline 13 & White throated bee-eater & Merops albicollis & 12 & $0.78 \%$ & 0.01 \\
\hline 14 & Dwarf kingfisher & Ispidina lecontei & 22 & $1.44 \%$ & 0.01 \\
\hline 15 & Black heron & Egretta ardesiaca & 21 & $1.37 \%$ & 0.01 \\
\hline 16 & Woolly-necked stork & Ciconia episcopus & 54 & $3.53 \%$ & 0.04 \\
\hline 17 & Narinas trogon & Apaloderma narina & 11 & $0.72 \%$ & 0.01 \\
\hline 18 & African thrush & Turdus pelios & 82 & $5.36 \%$ & 0.05 \\
\hline 19 & Laughing dove & Streptopelia senegalensis & 19 & $1.24 \%$ & 0.01 \\
\hline 20 & Red-neck dove & Streptopelia capicola & 35 & $2.29 \%$ & 0.02 \\
\hline 21 & Lemon dove & Aplopelia larvata & 25 & $1.63 \%$ & 0.02 \\
\hline 22 & Little swift & Apus afinis & 53 & $3.46 \%$ & 0.03 \\
\hline 23 & Mottled swift & Tachymarptis aequatorialis & 29 & $1.89 \%$ & 0.02 \\
\hline 24 & Hunter sunbird & Nectarinia hunter & 102 & $6.66 \%$ & 0.07 \\
\hline 25 & White crowned starling & Spreo albicapillus & 53 & $3.46 \%$ & 0.03 \\
\hline
\end{tabular}




\begin{tabular}{|l|c|c|c|c|c|}
\hline $\mathbf{2 6}$ & Square-taled drongo & Dicrurus ludwigii & 67 & $4.38 \%$ & 0.04 \\
\hline $\mathbf{2 7}$ & Slender- billed weaver & Ploceus pelzeini & 80 & $5.23 \%$ & 0.05 \\
\hline $\mathbf{2 8}$ & Black-billed water & Ploceus cucullatus & 16 & $1.05 \%$ & 0.01 \\
\hline $\mathbf{2 9}$ & Chestnut weaver & Ploceus ribiginosus & 12 & $0.78 \%$ & 0.01 \\
\hline $\mathbf{3 0}$ & Red handed weaver & Anaplectes rubriceps & 15 & $0.98 \%$ & 0.01 \\
\hline $\mathbf{3 1}$ & Compact weaver & Ploceus pachyrhnchus & 14 & $0.91 \%$ & 0.01 \\
\hline $\mathbf{3 2}$ & Red-chested swallow & Hirundo lucida & 61 & $3.98 \%$ & 0.04 \\
\hline $\mathbf{3 3}$ & Red-rumped swallow & Cecropis daurica & 46 & $3.00 \%$ & 0.03 \\
\hline $\mathbf{3 4}$ & Cape rook & Corvus capensis & 26 & $1.70 \%$ & 0.02 \\
\hline $\mathbf{3 5}$ & White wing starling & Neocichla gutturalis & 105 & $6.86 \%$ & 0.07 \\
\hline $\mathbf{3 6}$ & Fisher's starling & Spreo fischeri & 23 & $1.50 \%$ & 0.02 \\
\hline $\mathbf{3 7}$ & Senegal coucal & Centropus senegalensis & 34 & $2.22 \%$ & 0.02 \\
\hline $\mathbf{3 8}$ & Yellow-rumped seed-eater & Serinus atrogularis & 8 & $0.52 \%$ & 0.01 \\
\hline
\end{tabular}

Table 6: Mean number of birds species observed in the three land use types of Ilejemeje Local government area, Ekiti State.
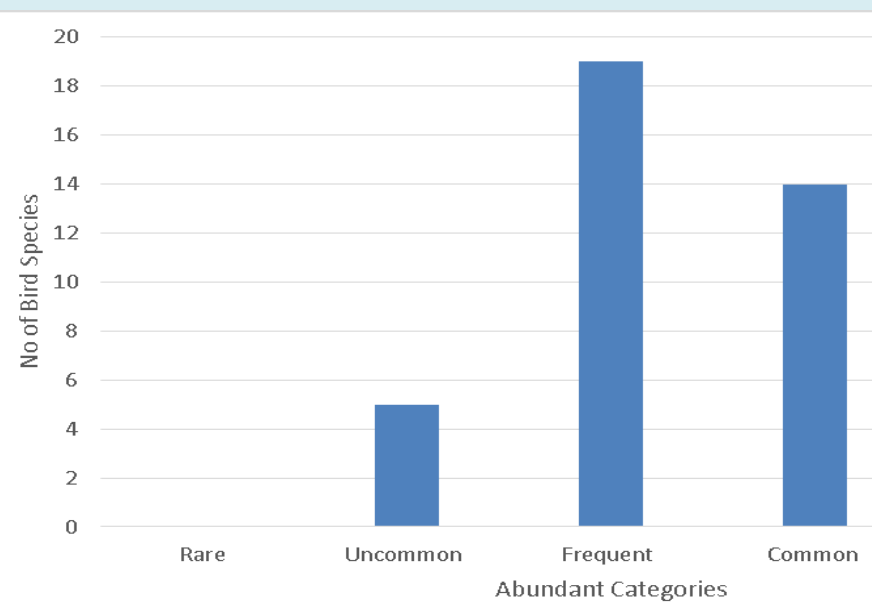

Abundant

Figure 3: Bird species in different relative abundance categories in the land use types of Ilejemeje LGA.

\section{Discussion}

A total number of thirty eight (38) bird species were recorded in the three land use types of Ilejemeje local government area which indicated that the area has a considerable number of bird species spread across the three (3) land use types. The abundance and species richness of bird species in the agricultural land use area especially maize plantation revealed highest among the three studied land use types as shown in Table 3 of the result. This shows that farming activities is an influencing factor on the bird species abundance and richness in the area. During the survey, more of species and individual birds were recorded in maize plantation than in cocoa and natural forest. The overall number of recorded bird species was highest in maize plantation (34 species), followed the natural forest
(25 species) and lastly by cocoa plantation (22). Also the abundance of individual bird species observed in the study area ranked highest in the maize plantation (637) followed by cocoa plantation (472) and lastly by that of natural forest (422). The distribution of bird species observed in the three land use types vividly revealed that the seventeen (17) of the birds recorded in the natural forest were completely absent in the maize plantation while ten (10) bird species exclusively recorded in maize plantation were absolutely absent in both the cocoa and natural forest as shown in table 5 . This finding adds to the evidence that tropical natural forest bird species are adversely affected by habitat modification.

This result is consistent with previous studies of [1317] that analyzed agricultural matrix showed evidence of supporting rich but modified bird communities. The higher 


\section{Journal of Ecology and Natural Resources}

number of bird species recorded in the maize plantation during the study may be due to the relative low nature species pool and the proportionally high number of open habitat specialists present in the area, despite the fact that natural forest had relatively lesser number of bird species and the least value of individual bird species abundance as compare to maize plantation, natural forest ranked fairly next higher in exclusive bird species to maize plantation. Also the avian orders, family and species composition in the three land use types of Ilejemeje local government area are not homogeneous as shown in table 2 .

This variation in avian orders, families and species composition may due to vegetation heterogeneity in the study land use types. The vegetation heterogeneity invariably brings about the variation in food, cover availability and micro-climatic variation. This agrees with the conclusion of Afolayan and Ajayi [17] and Crowel [19] that the variation in species composition, abundance and diversity of animal are strongly influence by the availability of food, water and cover in the habitat. Further analysis of bird species composition and relative abundance of the bird species of the area as shown in figure 3 indicated that out of thirty eight (38) bird species observed in the study area, five (5) bird species were uncommon, nineteen (19) birds were frequent, fourteen (14) bird species were common, while none of the species observed were in rare and abundant categories. The relative abundance of bird species in the local government area might be related to the availability of food, habitat condition and breeding season of the species. The distinct seasonality of rainfall and seasonal variation in the abundance of food resources result in seasonal change in the species abundance of birds [20,21].

The land use type distribution pattern of birds showed highest species richness which ranges from 2.78 to 4.02 and Shannon diversity index $\left(\mathrm{H}^{\prime}\right)$ (0.82 to 0.89$)$ in maize plantation, which could be due to the presence of sufficient food in form of seed and insect, while the species richness ranges from 1.62 to 3.64 and diversity index 0.59 to 0.86 occurred in cocoa plantation and that could be as a result of less availability of the food sources. The natural forest plantation had the species richness that ranges from 2.01 to 2.23 , Shannon diversity index 0.68 to 0.88 . The highest species evenness index (E) was registered in cocoa plantation. This is followed by that of natural forest plantation and maize plantation. This vividly showed that bird species were more evenly distributed in cocoa plantation than in natural forest and maize plantation. The state of micro-climate of the cocoa plantation as compare to the other land use types might be the contributive factor to the highest bird species evenness. This is because of the available of variety of alternative food source for bird; moreover, the natural forest plantation is less disturbed by people, thereby contributing to a favourable condition for breeding, and nesting sites. Whereas, maize and cocoa plantation are fragmented and expose to local people for cultivated and cutting of vegetation. As a result of this bird which depend on these sites for feeding, nesting and breeding, are affected. In natural habitat where the intervention of humans is less and minimum, the evenness of species is higher than the fragmented ones where intensive farming is carried out [22].

Different group of bird species seems to respond differently to feeding habits, ten feeding guilds were identify in respect to the food habits of bird species inhabiting the area. The feeding guilds, varies with the land use types as shown in fig. 2 of the result. Two bird species categories that belong to granivore-insectivore (GI), and frugivoreinsectivores (FI) ranked highest in the maize plantation. In cocoa plantation, insectivore (I) ranked highest, while granivore-insectivore (GI) ranked next to the highest feeding guild. Natural forest plantation had insectivore (I) as the highest feeding guild followed by frugivore- insectivore (FI) and granivore-insectivore (GI). The study vividly showed that a substantial shift occurred from specialist to generalist bird species in the studies area. This seems to indicate that generalist birds are more prevalent in the study area than the specialists since resources of specialist may be dwindling in the varying land use types. Elmquist [23] asserted that nature fragmented system and habitat disturbance leads to loss of specialist species and encourage the generalist.

The study shows that insectivore's bird species were more prominent in cocoa and natural forest plantations than in maize plantation, which was in agreement with the findings of Thiollay [13], Komar [24] and Waltert [16] which confirmed that in annual agricultural area, insectivores mean number of recordings per visit dropped by $50 \%$ in relation to forest controls. The Granivore- insectivore (GI) feeding guild were dominant in the three land use types and have no marked difference in the frequency of occurrence among the land use types. This insignificance in the frequency of occurrence might be due to the fact that a large number of trees whose seeds were edible to bird species and which has the characteristic of attracting insects were scattered across the landscape in both agricultural areas and natural forest plantation. The studies further showed that frugivoreinsectivore (FI) feeding guild showed variations in the frequency of occurrence among the three land use types as shown in figure 2 . The highest frequency was recorded in maize plantation, this is synonymous to the findings in other studies where frugivores seem to be the winners when it comes to land use changes. The result of study in table 6 further revealed the presence of seven (7) conspicuous relatively less shy and flocking species such as Black Kite (Milvus migrans), Double spurred francolin (Francolinus bicalcaratus), Slender-billed weaver (Ploceus pelzeli), Hunter 
Sunbird (Nectarinia hunteri), Africa Trush (Turchus pelios), White wing starling (Neocichia gutturdis).

\section{References}

1. Sala OE, Chapin FS, Armesto JJ (2001) Global biodiversity scenarios the year 2001. Science 287: 1770-1774.

2. Musters CJM, Graaf HJ, Keurs WJ (2001) Can protected areas be expected to be expanded in Africa. Science 287(5459): 1759-1760.

3. Tilman D, Fargione J, Wolff BD, Antonio C, Dobson A, et al. (2001) Forecasting agriculturally driven global environmental change. Science 292(5515): 281-284.

4. Raman TRS (2003) Assessment of census techniques for interspecific comparisons of tropical rainforest bird densities: a field evaluation in the western Ghats, India. Ibis 145(1): 9-21.

5. Naidoo R (2004) Species richness and community composition of songbirds in tropical forest agricultural landscape. Animal conservation 7(1): 93-105.

6. Chamberlain DE, Fuller RJ, Bunce RGH, Shrubb M (2000) changes in abundance of farmland birds in relation to the timing of agricultural intensification in England and Wales. Journal of applied Ecology 37(5): 771-788.

7. Benton TG, Bryant DM, Cole L, Crick HQP (2002) Linking agricultural practices to insect on bird populations: a historical study over three decades. Journal of Applied Ecology 39(4): 673-687.

8. Myers M (1998) Threatened Biotas: Hot spots in tropical forests. Environmentalis 8(3): 187-208.

9. Myers NEA, Mittermeicer A, Kent J (2000) Biodiversity hotspots for conservation priorities. Nature 403: 853858.

10. Bibby CJ, Burgess ND, Hill DA, Mustoe SH (2000) Bird census techniques. $2^{\text {nd }}$ (Eds.), London Academic Press.

11. Jones M (1998) Study design. Pp15-34. In: Bibby C, Jones $\mathrm{M}$ and Marseden S (Eds.), Expendition Field Techniques, Bird Surveys. Royal Geographical society with the Institute of British Geographer, London.

12. Borrow N, Demey R (2004) Birds of Western Africa, published by Christopher Helm an imprint of A and C Black publishers Ltd., 37 Soho square, London WSD-3QZ.
13. Thiollay JM (1995) The role of traditional agro forests in the conservation of rainforest birds in Sumatra. Conservation 9(2): 335-353.

14. Tejeda-Cruz C, Sutherland WJ (2004) Bird responses to shade coffee production. Animal Conservation 7(2): 169-179.

15. Sutherland WJ, Newton I, Green ER (2000) Bird Ecology and Conservation. A Handbook of Techniques. Oxford University Press, Oxford.

16. Waltert M, Bobo KS, Sainge MN, Fermon H, Muhenberg M (2005) From forest to farmland: Habitat effects on Afrotropical forest bird diversity. Ecological Applications 15(4): 1351-1366.

17. Harvey CA, Medina A, Sanchez DM, Vilchez S, Hernandez $B$, et al. (2006) Patterns of animal diversity in different forms of tree cover in agricultural landscapes. Ecological Applications 16(5): 1986-1999.

18. Afolayan TA, Ajayi SS (1980) The influence of seasonality on the distribution of large mammals in Yankari Game Reserve, Nigeria. Africa Journal of Ecology 18(1): 87-93.

19. Crowel CM, Schif JC, Gubb AA (1981) Effect of rainfall variation, fire vegetation and habitat physiognomy on Northern Animal Community. South Africa Wildlife resources 11(3): 87-104.

20. Gaston KJ, Blackburn TM, Goldewijk KK (2003) Habitat conversion global avian biodiversity loss. Proceedings of the Royal Society of London series B. Biological Sciences 270(1521): 1293-1300.

21. Karr JR, Roth RR (1971) Vegetation Structure and Avian Diversity in several New World Areas. American Naturalist 105(945): 423-435.

22. Rana SVS (2005) Essentials of Ecology and Environmental Science $2^{\text {nd }}$ eds. Prentice-Hall of India Private Ltd., New Delhi.

23. Elmquist TC, Folke M, Nystroem G, Peterson J, Bengtson B, et al. (2003) Response diversity ecosystem change and resilience. Frontiers in Ecological Environment 1(9): 488-494.

24. Komar O (2006) Ecology and conservation of birds in Coffee Plantations: a critical review. Bird Conservation International 16: 1-23. 